\title{
Esbozo de la educación ambiental en el currículum de educación básica en México. Una revisión retrospectiva de los planes y programas de estudio Outline of the Environmental Education Mexican Basic Education Curricula.
} A Retrospective Review of the Study Plans and Programs

\author{
Esperanza Terrón Amigón \\ Universidad Pedagógica Nacional, Ajusco, México
}

eterron@upn.mx

\section{RESUMEN}

El objetivo es presentar una interpretación retrospectiva sobre cómo fue promovida la educación ambiental (EA) en el nivel básico a partir del Programa de Modernización Educativa 1989-1994, resaltando sus particularidades y retos para poder pensar una transformación sustantiva en el momento actual. Como recurso metodológico se recurre a un ejercicio de análisis documental descriptivo y cronológico de las políticas y principales acciones implicadas en la institucionalización curricular de la EA en la educación básica, ya que apelar a la memoria histórica permite comprender qué hemos hecho, qué necesitamos cambiar y hacia dónde queremos ir. Se revisan documentos sobre la política educativa, planes y programas de estudio y los estados del conocimiento en EA en México. Las categorías de análisis que cruzan el texto son los enfoques: educativo, curricular, epistemológico, pedagógico, metodológico y ético. Se identifica que no basta que la EA se haya convertido en un campo de estudio, de desarrollo conceptual y de intervención educativa; es necesario reorientar su sentido en el currículo y sus formas de intervención, formar a los docentes y abrir un espacio curricular propio para la EA con una perspectiva de desarrollo de pensamiento fundado en la complejidad y la transdisciplinariedad inherentes a la EA.

PALABRAS CLAVE: educación ambiental, currículum, educación básica, enfoque educativo, formación de profesores, México

\section{ABSTRACT}

The objective is to present a retrospective interpretation of how environmental education (EE) was promoted at the basic level from the 1989-1994 Educational Modernization Program, highlighting its particularities and challenges in order to think about a substantive transformation at the present time. As a methodological resource we resort to a descriptive and chronological documentary analysis of the policies and main actions involved in the curricular institutionalization of EE in basic education, since appealing to historical memory allows us to understand what we have done, what we need to change and where we want to go. We review documents on educational policy, plans and curricula, as well as the EE states of knowledge in Mexico. The categories of analysis that cross the text are the educational, curricular, epistemological, pedagogical, methodological and ethical approaches. It is identified that it is not enough that the EE has become a field of research, of conceptual development and of educational intervention. It is necessary to redirect its meaning in the curriculum and its forms of intervention by training teachers and by opening their own curricular settings for the $\mathrm{EE}$ with a perspective of development of thought based on complexity and a transdisciplinary approach, inherent in EE.

KEY WORDS: environmental education, curriculum, basic education, educational approach, teacher upgrading, Mexico 


\section{INTRODUCCIÓN}

En este momento coyuntural que se vive en México, cuando se habla de impulsar una cuarta transformación de la República, repensar y cambiar la forma en que se ha desarrollado la EA en el currículo de educación básica es dar un paso adelante en educación; de lo contrario, no se estaría ofreciendo una educación integral en ese nivel educativo, ni se estaría respondiendo a la formación educativa indispensable en este siglo XXI. La EA tiene el imperativo de comprender la complejidad del problema ambiental planetario, de pensar nuevas formas de aprender y de enseñar, que sean reflexivas y críticas, y se sustenten en un compromiso ético y social que posibilite la reestructuración de la convivencia planetaria que degrada el ambiente, así como la mejora de la calidad de vida y del mundo en el que vivimos.

La educación básica tiene el potencial de apoyar en la construcción de principios que ayuden a que los estudiantes opten por un desarrollo profesional con responsabilidad ética, por relaciones personales y sociales con sentido humano, por valores ambientales e interculturales fundamentados en el cuidado, el respeto y la responsabilidad planetaria. Sin embargo, la educación básica es un trayecto formativo cuya importancia y complejidad no han sido suficientemente reconocidas, no obstante que en este nivel se erigen bases importantes del conocimiento y formación humanos, que posibilitarán a los estudiantes desarrollar potencialidades para moverse en el mundo con consciencia crítica, así como para transitar a la universidad con un sustento sólido que les impida rezagarse en el camino.

En México, la educación básica es obligatoria. A partir de la reforma de 1993, el periodo para cursarla se amplió a 11 años: dos para preescolar, seis para primaria, y tres para secundaria (SEP, 2009); la edad de los niños en el primer nivel va de tres a cinco ańos; en primaria, de seis a 12 ańos; y, en secundaria, de 12 a 15 años. Según cifras de la Secretaría de Educación Pública (SEP), para el ciclo escolar 2016-2017, de 1917237 niños que egresaron de la educación primaria, ingresaron a la educación secundaria sólo 903389 ; de éstos, sólo llegaron 642383 a la educación media superior y 433580 , a la educación superior (SEP, 2017b).

Ante ese panorama, repensar un proyecto educativo de nación compromete la reflexión sobre las cuestiones urgentes, que necesitan 
abordarse de manera sistémica en México para evitar el abandono escolar y favorecer el logro educativo en todos sus niveles y contextos, considerando los múltiples factores que concurren en esos problemas y las condiciones socioculturales en las que se desenvuelve la vida de los estudiantes, incluso dentro del seno familiar. Esto requiere no pasar por alto ni restar relevancia y urgencia a los niveles básicos, pues son el cimiento que permitirá consolidar elementos que posibiliten crear las condiciones factibles para generar los cambios que necesita en sus distintos contextos un país como México, diverso en lo ambiental, lo cultural, lo social y lo económico.

La EA, desde el nivel educativo básico, puede ser un potencial para la formación humana, ambiental e intercultural de los estudiantes y para transformar el mundo en que vivimos, en cuanto integra los elementos de la formación que se demandan en este siglo, ante la urgencia de forjar un futuro planetario más armónico y de paz.

En los planes de estudio de educación básica se alude el compromiso de formar sujetos críticos, capaces de comprender la complejidad del mundo, sus interconexiones e impactos, lo que también es un propósito de la EA por la condición planetaria, compleja y sistémica del problema donde, de acuerdo con Morin y Delgado (2016) y Leff (2015), concurren interacciones naturales y sociales que involucran factores cognitivos, económicos, políticos e ideológicos. Pese a ello, en las reformas y sus contenidos, a la EA -cuyo proceso de conocimiento requiere una reflexión integradora de esos factores, así como un enfoque transdisciplinar para encontrar soluciones- se le ha enmarcado en el dominio del conocimiento de las ciencias naturales, se le ha cerrado a la crítica de las causas sociales e históricas del problema y, como se verá en adelante, poco a poco se le ha empobrecido en los cambios curriculares posteriores a la Reforma del 1993.

Sin embargo, la EA es un campo de conocimiento social necesario en la educación de los nińos y jóvenes de nuestro país, entre otras cosas, debido a los graves problemas de degradación de nuestros sistemas ecológicos, las situaciones ambientales de riesgo nacional y planetario, como el cambio climático, la contaminación en general, la deforestación por la minería a cielo abierto, así como las enfermedades derivadas de tales situaciones que afectan, principalmente, en la población vulnerable de nuestro país. 
De ahí la importancia de recuperar la historia sobre cómo la EA se ha desarrollado en el nivel básico, así como de resaltar sus particularidades y retos como elementos de partida en la edificación de una transformación sustantiva de su práctica, sobre todo en este momento, que nos demanda transformar el afán materialista occidental del bienestar, profundizar en la humanización de las nuevas y las viejas generaciones, así como abrir vías que salven a la humanidad del desastre que la amenaza y que posibiliten mejorar las relaciones entre los humanos, y entre éstos y la Tierra.

A lo largo del artículo se identifican los principales rasgos que han singularizado el desarrollo de la EA. Entre sus logros, se destaca la inserción del estudio de problemas ambientales en algunas asignaturas curriculares, la formulación y desarrollo de cursos con docentes, la producción de libros, artículos y materiales educativos. Por otro lado, entre los retos, se señala la necesidad de superar la orientación positivista y técnica arraigada en el currículo, debido a que ésta se traduce en prácticas instrumentales, dispersas, desarticuladas y mecanicistas que debilitan, distorsionan y revierten el logro de la formación pretendida mediante la EA y la educación general.

Se concluye que no basta que la EA se haya convertido en un campo de investigación, de desarrollo conceptual y de intervención educativa, sino que es preciso reorientar su sentido en el currículo y sus formas de intervención, formar a los docentes y abrir un espacio curricular propio de la EA, con sustento epistemológico, pedagógico y ético, así como con una perspectiva de desarrollo de pensamiento fundado en la complejidad y la transdisciplinariedad inherentes a dicha educación. Esto aseguraría el aprendizaje y el gusto por el conocimiento disciplinar, pero también otras formas de aprender y transformar la realidad aprovechando, en términos de Olivé (2015), aportes de diferentes tipos de conocimientos -locales, tradicionales, científicos y tecnológicos-indispensables para comprender, cambiar y afrontar el mundo complejo en el que vivimos, en permanente interacción e incertidumbre.

El artículo aborda los siguientes puntos: surgimiento de la educación ambiental; introducción de la educación ambiental en el currículo; investigación en educación ambiental; controversias en la práctica de la educación ambiental, y conclusiones. 


\section{SURGIMIENTO DE LA EDUCACIÓN AMBIENTAL}

La Educación Ambiental (EA) aflora en un contexto de múltiples tensiones y en un clima de renovación y de cambios en todos los ámbitos de organización social, catapultados por los movimientos ecologistas, ambientalistas, estudiantiles y contraculturales ante el conjunto de crisis mundiales que emergieron en los últimos 50 ańos del siglo XX, así como en el marco de circunstancias coyunturales internacionales.

La mundialización de la crisis en los órdenes ambiental, económico, energético, alimentario, militar y espiritual, entre otros problemas del mundo que, "por primera vez en la historia de la humanidad se juntan de manera explosiva y catastrófica”, aunada a la globalización de los mercados y de los medios de comunicación, reveló que la crisis ambiental no se trataba de una, entre otras, sino de una crisis de la evolución del modelo civilizatorio predominante en los últimos doscientos o trescientos ańos, caracterizada por la ideología del "desarrollismo", del consumismo y la aceleración de la economía ficticia especulativa (Estermann, 2012, p. 151).

A través de un proceso largo de reflexión y en el centro de un conjunto de cambios y movimientos sociales que permearon los ańos sesenta del siglo XX, se creó la EA con el propósito de atender la necesidad social de enfrentar y de reencausar el sentido de la vida, los valores materialistas, consumistas y despilfarradores que mantienen e intensifican la crisis ambiental en el planeta, cuya complejidad es creciente.

Los fines, objetivos, métodos y principios educativos de la EA -que era necesario impulsar para contribuir en la transformación de los valores y relaciones humanas que inciden en la crisis ambientalse formalizaron institucionalmente en 1977, durante la Conferencia Intergubernamental de EA llevada a cabo en Tbilisi, Georgia, antes Unión de Repúblicas Soviéticas Socialistas (URSS), convocada por la Organización de las Naciones Unidas para la Ciencia la Cultura y la Educación (UNESCO).

La evolución histórica de la educación, en lo que respecta a la EA, dio un giro trascendente en esa conferencia pues, ante la evidencia de la complejidad de la crisis ambiental y su gravedad, se le confirió un enfoque educativo crítico, complejo e interdisciplinario, diferente del de la racionalidad capitalista (instrumental, lineal, par- 
celada), al reconocer que tal crisis es resultado de la interacción de la sociedad con la naturaleza y que en esa interacción concurren aspectos biológicos, físicos, sociales, económicos, políticos, culturales y educativos (UNESCO, 1980; González, 1997; Leff, 2003, y Terrón, 2004, entre otros).

La EA se crea como una alternativa para contribuir en la transformación social, y emerge con un enfoque propio de construcción de conocimiento que otorga un lugar importante a la toma de conciencia reflexiva del sujeto sobre el problema ambiental global y sus correlaciones, así como frente al imperativo de forjar valores que posibiliten cambios en las relaciones humanas que dańan el ambiente y ponen en riesgo la vida en el planeta. En términos simples, se busca aprender de manera diferente y de actuar y relacionarnos de otra forma.

En el marco del clima de renovación y de cambios que demandaba la época, la EA se constituyó como un modelo educativo crítico, complejo e interdisciplinario, alternativo al modelo instrumental técnico prevaleciente, en tanto que superar la crisis ambiental exigía nuevas formas de comprensión del mundo, sustentadas en nuevas epistemologías - más allá de los modelos positivistas- y en una ética del respeto, y de cuidado a la Tierra y a la vida, así como en relaciones armónicas responsables entre los humanos y entre las culturas.

De cara a los valores que inducen a la degradación ambiental, mediante la EA se plantea un proyecto civilizatorio que busca cambiar el paradigma de la vida ponderando el bien común; se pretende una vida no materialista ni consumista, sino el bienestar social planetario, la justicia social y económica, modos de vida sustentables, el respeto a la diversidad cultural y a los patrones tradicionales de subsistencia que veneran y honran a la Tierra y a sus sistemas ecológicos de apoyo a la vida, todo con base en el diálogo de saberes y relaciones horizontales, como posibilidades para garantizar la convivencia planetaria y una cultura de paz (UNESCO, 1977 y 1980; Leff, 2015; Terrón, 2017, entre otros).

De cara a los procesos que mostraron las limitaciones del conocimiento y de los valores hegemónicos instrumentales frente a la tensión de la crisis ambiental, los elementos filosóficos, epistemológicos, conceptuales, metodológicos, educativos y éticos con los que 
se dota a la EA señalan nuevos senderos para el desarrollo humano. Se trata de asumir miradas múltiples en busca del conocimiento y su reorganización, para contribuir a la toma de conciencia crítica sobre el problema ambiental con visión compleja, holística, histórica, relacional, inter y transdisciplinar. La EA busca en la ética no sólo un freno a las conductas que degradan el ambiente, sino un proceso de renovación profunda en las relaciones sociales que dañan la convivencia planetaria, lo que involucra un cambio cultural en la forma de concebir el mundo, la ciencia, la naturaleza y la vida.

En México, la incorporación oficial de la EA al sistema educativo del nivel básico se puede ubicar en la Reforma educativa de 1993 y, concretamente, en el Programa Nacional para la Modernización Educativa 1989-1994 (PROMODE). Ahí se estableció la incorporación de contenidos sobre la dimensión ambiental con base en tres ejes: 1) los problemas ambientales; 2) alternativas para prevenirlos y disminuirlos, y 3) valores para una mejor convivencia social. Entre éstos se destacaban los valores del respeto mutuo, la responsabilidad, la equidad y la colaboración, entre otros (Poder Ejecutivo Federal y SEP, 1989).

Con todo, el modelo educativo producto de la Reforma de 1993 cambió de un enfoque centrado en la enseñanza, a uno centrado en el aprendizaje de los alumnos, y de un modelo curricular por áreas a otro por asignaturas, lo cual debilitó la introducción de la EA en el currículo, frente al reto de entender la compleja realidad ambiental y de forjar nuevas formas de pensar el mundo para un actuar consciente e integral en la solución de los problemas ambientales.

La política educativa nacional recogió la demanda internacional de incorporar transversalmente la EA en el currículo de los diversos niveles educativos y, a partir de la reforma de 1993, con diversas reformulaciones, esa política aún orienta la educación del país. En las subsecuentes reformas a la educación básica se ha mantenido el discurso sobre el cuidado y protección del ambiente, la conservación de la naturaleza y el uso racional de los recursos naturales. 


\section{INTRODUCCIÓN DE LA EDUCACIÓN AMBIENTAL EN EL CURRÍCULO}

En México, la entrada de la política nacional para el ambiente tiene varios momentos y matices; se delinea en el Plan Nacional de Desarrollo 1983-1988; en el caso de la educación, esa política se respalda en el Decreto Presidencial del 14 de febrero de 1986, cuyo artículo $5^{\circ}$ instruye a la SEP a fin de que tome medidas pertinentes para iniciar una pedagogía ecológica formal a nivel nacional (De Alba, en Terrón, 2004). Tal instrucción consistió en: a) introducir la materia de ecología en los planes de estudio de los maestros; b) incorporar contenidos educativos de temas ecológicos en los libros de texto de los diferentes niveles escolares; c) realizar programas de capacitación para el magisterio en esa materia, y d) propiciar que el servicio social de las licenciaturas se oriente a temas ecológicos. ${ }^{1}$

Derivado de tales disposiciones, tres ańos después se suscribieron esas recomendaciones en el Programa Nacional para la Modernización Educativa 1989-1994 (Promode); asimismo, la Ley General de Educación, publicada en 1993, de acuerdo con el decreto presidencial y con el Promode, estableció que la educación básica debía "inculcar el respeto a los derechos humanos, la protección de los recursos naturales y el medio ambiente" (SEP, 1993a, p. 38). En las reformas realizadas a dicha Ley en 2002 y 2011, permanecen entre los fines de la educación el de "inculcar la valoración de la protección y conservación del medio ambiente como elementos esenciales para el desenvolvimiento armónico e integral del individuo y la sociedad" (Ley General de Educación, 1993, p. 3. Última Reforma DOF 19-01-2018).

En el Programa de Desarrollo Educativo 1995-2000 (Poder Ejecutivo Federal, 1996), se sostuvo lo que el Promode y la Ley Federal de Educación respaldaron respecto al ambiente. Ello se puede identificar en los aspectos que, de acuerdo con el Promode (Poder Ejecutivo Federal y SEP, 1989), la educación básica tenía que garantizar en sus planes y programas, entre éstos:

\footnotetext{
${ }^{1}$ Para ampliar la información sobre la fase central de la entrada de dicha política, consúltese Terrón, 2004.
} 
- Crear conciencia en los estudiantes de la gravedad del problema ecológico; de que vivimos en un planeta de recursos limitados y frágiles, y de la necesidad de adoptar conductas que favorezcan su equilibrio y mejoramiento.

- La adquisición de conocimientos para comprender los fenómenos naturales, proteger el ambiente y usar racionalmente los recursos naturales; el fomento de valores y actitudes que cristalicen en conductas democráticas, que contribuyan al respeto de los derechos humanos en todos los ámbitos de la vida social y de convivencia; una práctica educativa que sea la expresión de valores de la democracia, la honradez, el aprecio por el trabajo y por los que trabajan y el sentido de pertenencia a una gran nación, su historia y su cultura.

- Una vida escolar que refuerce la autoestima, el respeto, la tolerancia, la libertad para expresarse, el sentido de responsabilidad, la actitud cooperativa, entre otros. En las ciencias naturales, fomentar el amor por la naturaleza, el entendimiento de cuestiones significativas para la calidad de vida, la preservación de la salud, el desarrollo personal equilibrado, la protección y la preservación de los recursos y del medio ambiente.

- El desarrollo armónico con la naturaleza y la sustentabilidad en el proceso de formación de la sociedad mexicana tienen calidad de prioridad en el Plan Nacional de Desarrollo 2001-2006 (Poder Ejecutivo Federal, 2001, pp. 41-42; 73; 91-92; 122-125).

De acuerdo con ello, en el subprograma de educación básica la SEP explicita la necesidad de fortalecer en el currículo los contenidos de la EA y para el desarrollo sustentable (SEP, 2001, p. 144).

\section{Diagnóstico: educación ambiental, contenidos y necesidades educativas}

El Promode fue un motor clave en el desarrollo institucional de la EA; en su base se encuentra la realización previa de un diagnóstico que incluyó La Consulta Nacional para la Modernización de la Educación, ejecutada por el Consejo Nacional Técnico de la Educación (Conalte) en los primeros meses de 1989; el resultado de la consulta “estableció como prioridad la renovación de los contenidos y los mé- 
todos de enseñanza, el mejoramiento de la formación de maestros y la articulación de los niveles educativos" (SEP, 1993b, p. 11). En el caso de la educación básica, se llevaron a cabo tres investigaciones que buscaban aportar elementos sobre las prioridades y necesidades educativas relativas al ambiente y sobre el estado de la EA en los niveles preescolar, primaria y secundaria. Se analizaron: el currículo, los programas y los libros de texto correspondientes a las reformas de 1972 y de 1974, vigentes en aquel momento (Terrón, 2004).

En educación preescolar, la investigación de Chamizo (1990) Análisis de los contenidos de educación ambiental en los Programas de Educación Preescolar, 1986-1987 subraya que la EA es escasa en los programas que analizó de diversos contextos: urbano, rural e indígena, excepto en el programa del Conafe. Asimismo, destacó como grave trabajar por líneas durante todo el año escolar, considerando que el problema ambiental se resuelve incluyendo una unidad sobre ecología o mediante acciones informativas que se agotan en la descripción de fenómenos ajenos a la realidad social del niño, en cuanto que la EA no es un problema de contenidos a aprender, sino una concepción ante la vida. También prestó relevancia al hecho de que se estaba perdiendo de vista el valor que tendrá el ambiente, cuando el educando sea mayor, pues encontrará sus raíces en la formación que sobre ello se le ofreció en su niñez.

En educación primaria, de Alba et al. (1993b), en su estudio Dimensión ambiental en el currículum de la escuela primaria, 1985-1986, resaltan una gran riqueza de contenidos ecológicos, pero recomiendan enriquecerlos en materia ambiental, clarificar la noción de ciencia, método y validez científica desde donde se mira la dimensión y problemática ambiental, así como esclarecer desde dónde se aborda el tratamiento de los contenidos ambientales, tanto en ciencias naturales como en ciencias sociales.

En la educación secundaria, Jiménez y Hernández (1988) analizaron la EA en los libros de texto, y destacaron que los contenidos de los libros presentaban una incapacidad instrumental y teórica para develar las situaciones ambientales vinculadas no sólo con las condiciones de desarrollo del hombre, sino con la complejidad de la ecología y el origen de la cultura, en cuanto ésta se encuentra en la base de las múltiples interrelaciones y adaptaciones que el hombre ha construido con la naturaleza, amén de que la fragmentación y 
parcelación del conocimiento en los contenidos ambientales obstaculizaba una visión de conjunto de la dimensión ambiental.

Los resultados de las investigaciones señaladas son muy similares a los obtenidos en la Consulta Nacional para la Modernización de la Educación, que se enlistan a continuación. Éstos también concuerdan con observaciones críticas que, desde la Conferencia de Estocolmo, en1972, ya se hacían a ciertas formas de abordar la EA.

La Consulta Nacional para la modernización de la Educación Básica que realizó el Conalte (1992), arrojó los resultados siguientes: ${ }^{2}$

- Carencia de una cultura ecológica entre los educandos y los adultos, que se refleja en su falta de valores y de respeto hacia la naturaleza, lo que se atribuye al enfoque, organización y desarrollo de los contenidos, métodos y materiales educativos aplicados en las décadas anteriores a la consulta.

- Un enfoque informativo y memorístico, más que formativo, de los temas ecológicos, que inhibe el desarrollo de valores y actitudes de respeto y preservación del medio en el educando. Métodos y procedimientos didácticos que no favorecen el desarrollo de un pensamiento crítico, creativo y participativo, y tampoco estimulan en el educando el interés por la investigación.

- Contenidos sobre aspectos ecológicos que se abordan de manera aislada y fragmentada en los programas y libros de texto, y que carecen de un tratamiento interdisciplinario integrado, como se encuentran los componentes de la naturaleza y programas de ciencias naturales con los contenidos necesarios para que el alumno adquiera los conocimientos básicos sobre el medio natural, pero no ofrecen una propuesta de EA, porque la perspectiva desde la que se abordan deja fuera del análisis las relaciones que las sociedades establecen con el medio ambiente y las tecnologías que han empleado para aprovechar sus recursos. Los hallazgos de la consulta nacional y de las investigaciones

\footnotetext{
${ }^{2}$ Los datos mostrados sobre el diagnóstico tienen su fuente en una ponencia suscrita por el CONALTE en la memoria del Foro Internacional para la Incorporación de la Dimensión Ambiental en la Educación Media Superior, celebrado en la ciudad de Aguascalientes del 7 al 11 de junio de 1992. Ya que esta información se conoce poco, consideré importante plasmar lo que, al parecer, es la única información oficial sobre el enfoque de EA que sirvió como marco de referencia en la elaboración de los programas emergentes 1992-1993, de los planes y programas de estudio de la educación básica y de los libros de texto gratuitos de educación primaria (CONALTE, 1992; en Terrón, 2010).
} 
previas a la consulta fueron parte del proceso de diagnóstico realizado para identificar los principales problemas educativos del país, así como los contenidos indispensables para la formación de las nuevas generaciones. Entre esos contenidos se encuentran: la aplicación de criterios racionales en la utilización de los recursos naturales y la protección del ambiente y su protección, considerados más tarde en los planes y programas de estudio de la Reforma Educativa de la Educación Básica, 1993 (SEP, 1993b).

\section{Enfoque de la educación ambiental y de los planes de estudio de educación básica}

Para poner en contexto el trayecto de la política educativa y tener una visión de conjunto del proceso que conlleva el posicionamiento de la EA en la educación básica se presenta el cuadro 1, donde se concentran los eventos referidos líneas atrás hasta su consolidación en la reforma de los planes y programas que se han realizado hasta la fecha.

Como puede observarse, en la parte baja del cuadro 1, la etapa iniciada con la Consulta Nacional y las investigaciones en el nivel básico dieron como resultado el Promode 1989-1994; la prioridad inicial de este programa se concretó con la renovación de los contenidos y los métodos de enseńanza en la Reforma de 1993 del nivel primaria; su tercera prioridad, la articulación de los niveles educativos que conforman la educación básica, "Reforma Integral de la Educación Básica (RIEB)”, inició con la Reforma del nivel de preescolar, en 2004; continuó con la Reforma en educación secundaria, en 2006, y cerró con la educación primaria, en 2009. El objetivo de la RIEB fue concretar la articulación curricular de esos tres niveles educativos, incluso con la reforma integral de la educación media superior (RIEMS), un propósito previsto desde la reforma de 1993 (SEP, 1993a y 2009). 
CUADRO 1. Momentos relevantes que suscriben la incorporación de la dimensión ambiental en la Educación Básica

\begin{tabular}{|c|c|c|c|c|c|}
\hline 1986 & $1986-1989$ & 1989 & 1991 & 1992 & 1993 \\
\hline $\begin{array}{l}\text { Decreto Presidencial } \\
\text { del } 14 \text { de febrero } \\
\text { de } 1986 \text {, Artículo } 5^{\circ} \\
\text { (De Alba, en Terrón, } \\
2004 \text { ) }\end{array}$ & $\begin{array}{l}\text { Diagnóstico } \\
\text { Plan Nacional } \\
\text { de Desarrollo } \\
\text { 1989-1994 (SEP, } \\
\text { 1993b) }\end{array}$ & $\begin{array}{l}\text { Programa de } \\
\text { Modernización } \\
\text { Educativa 1989- } \\
1994 \text { (Poder } \\
\text { Ejecutivo Federal y } \\
\text { SEP, 1989) }\end{array}$ & $\begin{array}{l}\text { CONALTE "Nuevo } \\
\text { Modelo Educativo" } \\
\text { Propuesta para la } \\
\text { Modernización de } \\
\text { la Educación Básica } \\
\text { (SEP, 1993b) }\end{array}$ & $\begin{array}{l}\text { Acuerdo } \\
\text { Nacional para la } \\
\text { Modernización } \\
\text { Educativa (SEP, } \\
\text { 1993b) Inicio } \\
\text { de la Última } \\
\text { etapa, delinea las } \\
\text { orientaciones para } \\
\text { la transformación de } \\
\text { los planes de estudio }\end{array}$ & $\begin{array}{l}\text { Ley General } \\
\text { de Educación } \\
\text { Pública, } 1993\end{array}$ \\
\hline $\begin{array}{l}\text { Reforma Educación } \\
\text { básica primaria }\end{array}$ & \multicolumn{3}{|c|}{$\begin{array}{l}\text { Reforma Integral de la Educación Básica (RIEB) -Enfoque de } \\
\text { competencias -Estándares de desempeño: conocimientos, } \\
\text { habilidades, actitudes y valores -Aprendizajes esperados } \\
\text {-EA eje transversal (SEP, 2006) }\end{array}$} & $\begin{array}{l}\text { Propuesta RIEB } \\
\text { "Integra" las nuevas } \\
\text { formulaciones } \\
\text { hechas durante el } \\
\text { proceso de la RIEB }\end{array}$ & $\begin{array}{l}\text { Aprendizajes } \\
\text { clave para la } \\
\text { educación } \\
\text { integral }\end{array}$ \\
\hline 1993 & 2004 & 2006 & 2009 & 2011 & 2017 \\
\hline $\begin{array}{l}\text { Plan y programas de } \\
\text { estudio, y libros de } \\
\text { texto (SEP, 1993b) }\end{array}$ & $\begin{array}{l}\text { Educación } \\
\text { Preescolar } \\
\text { Programa (SEP, } \\
\text { 2004) }\end{array}$ & $\begin{array}{l}\text { Educación } \\
\text { Secundaria Plan } \\
\text { y programas de } \\
\text { estudio, y libros de } \\
\text { texto (SEP, 2006) }\end{array}$ & $\begin{array}{l}\text { Educación Primaria } \\
\text { Plan y programas } \\
\text { de estudio, y libros } \\
\text { de texto (SEP, } \\
2009 \text { ) }\end{array}$ & $\begin{array}{l}\text { Plan de estudios } \\
\text { 2011. Educación } \\
\text { Básica (SEP, 2011) }\end{array}$ & $\begin{array}{l}\text { Plan y } \\
\text { programas } \\
\text { de estudio, y } \\
\text { libros de texto } \\
\text { de } 1^{\circ} \text { y } 2^{\circ} \\
\text { Grados (SEP, } \\
2017 a \text { ) }\end{array}$ \\
\hline
\end{tabular}

Fuente: elaboración propia.

Las necesidades de formación que en el Promode -resultado de la etapa de consulta- fueron consideradas como indispensables para fortalecer los contenidos de la nueva Reforma de la educación básica son tres: "la renovación de los contenidos y los métodos de enseñanza, el mejoramiento de la formación de maestros y la articulación de los niveles educativos que conforman la educación básica”, lo cual se expresó en el Acuerdo para la Modernización Educativa 1992, y está en la base de las orientaciones que han servido de fundamento a la Reforma de 1993 y a las subsecuentes (SEP, 1993b, p. 11).

Entre las orientaciones que se priorizan en los planes y programas de estudio se encuentran las siguientes:

- La adopción de un enfoque formativo, constructivista y por competencias (para el aprendizaje permanente, el manejo de in- 
formación y de situaciones para la vida, para la convivencia y la vida en sociedad).

- En la asignatura de español, primer nivel de prioridad, el dominio de la lectura, la escritura y la expresión oral.

- En la asignatura de matemáticas, el desarrollo del pensamiento matemático a partir de situaciones prácticas.

- En ciencias naturales, atención especial a los conocimientos relacionados con la preservación de la salud, la nutrición, la protección del ambiente y el uso racional de los recursos naturales. Estos contenidos se amplían al conjunto de las actividades escolares y a las asignaturas de geografía y educación cívica.

- En educación cívica, principios éticos que preparen a los estudiantes en el conocimiento de sus derechos y deberes y en la práctica de valores para su vida personal.

- Esto supone conocer las características de la identidad nacional y el alcance de los derechos y obligaciones del individuo, así como una primera información sobre la organización política y las instituciones del país. Una educación básica procura, también, un nivel cultural afín a nuestra civilización y a la historia nacional, y forma la personalidad fundándola en valores como la honradez, el respeto, la confianza y la solidaridad, que son indispensables para una convivencia pacífica democrática y productiva (Diario Oficial de la Federación, 1992, p. 7).

- En educación física y artística, el desarrollo de actitudes propicias para el aprecio y disfrute de las artes y el ejercicio físico y deportivo.

Tales contenidos son el eje de la transformación de los planes y programas de estudio de la educación básica en todas sus Reformas, la de 1993, la RIEB (2004, 2006, 2009), el plan 2011, que integra las nuevas formulaciones incorporadas en las diferentes etapas de la RIEB y, el plan 2017, que busca fortalecer esos ejes ante los nuevos retos del mundo complejo y de cambios vertiginosos que vivimos. Para ello, reafirma la necesidad de formar sujetos reflexivos, participativos, con capacidad de lograr su desarrollo personal, laboral y familiar y dispuestos a participar en la solución de problemas naturales y sociales. El plan precisa los aprendizajes clave indispensables para la educación integral del siglo XXI, confirma el enfoque huma- 
nista, socioconstructivista y los métodos de aprendizaje cooperativo y colaborativo.

Cabe destacar que, en este artículo, se da seguimiento a lo relativo a la EA y, en este sentido, este ámbito -aunque en un principio no se nombra como tal- se consideró un campo formativo indispensable para fortalecer los contenidos de la educación básica, por lo que se le enmarca en el siguiente propósito:

- la protección del ambiente, el uso racional de los recursos naturales o protección de los recursos naturales.

Es un eje que, con diferente nivel de profundidad, se encuentra en la asignatura de ciencias naturales de todos los niveles educativos de la educación básica, en geografía y educación cívica. En la reforma del nivel secundaria se ubicó a la EA como Eje transversal (SEP, 2006); es el plan en el que se le otorgó un espacio para especificar en qué consiste, con una visión que rescata su complejidad y su carácter crítico. Posteriormente, en educación básica primaria, se fortaleció el modelo educativo por competencias; un logro esperado en el perfil de egreso de este nivel es que los estudiantes asuman el cuidado de la salud y del ambiente como condiciones que favorecen un estilo de vida activo y saludable (SEP, 2009).

Más adelante, en el plan 2011, que resume las reformulaciones del proceso de la RIEB, se menciona poco a la EA, sin dejar de otorgar vigencia a los problemas ambientales y a la protección de la naturaleza (SEP, 2011). En la reforma de 2017, la EA se sigue centrando en los programas de ciencias (SEP, 2017a), geografía y educación cívica; pero, los aprendizajes esperados se limitan a que los estudiantes hayan aprendido a identificar los problemas ambientales locales y globales, a cómo cuidar el medio natural y acciones para mitigar problemas.

Entre los aspectos relevantes de las potencialidades y limitaciones del enfoque ambiental que se adopta en los planes de estudio de la Reforma de 1993, y que no han cambiado en las subsecuentes reformas, pueden distinguirse los siguientes:

En el programa de educación preescolar, una potencialidad es la aproximación a la realidad desde una visión global, para fortalecer el propósito de interesar al nińo en los fenómenos naturales y en el cui- 
dado y preservación del medio ambiente; con tal fin, se creó el espacio de la naturaleza dentro de las aulas, un bloque de juegos y actividades denominado Relación con la naturaleza. Los temas que ahí se abordan se enfocan en la ecología, la salud y la ciencia (SEP, 2004). La limitante es que el énfasis se pone sólo en el medio natural, sin reflexionar sobre la participación social y sus valores en el problema.

En educación primaria, el enfoque globalizador de la EA se aplicaría muy bien en sus primeros dos grados, ya que en un sólo texto se integran las asignaturas de ciencias naturales, geografía, historia y educación cívica; sin embargo, se soslaya el enfoque globalizador, pese a la recomendación de utilizar como elemento articulador el conocimiento del medio natural y social que rodea al niño.

De igual manera, el potencial de las asignaturas integradas modularmente en los primeros dos grados de educación primaria se pierde en el tercer grado, debido a que esas asignaturas se separan $y$ adoptan un enfoque lineal y parcelado. La asignatura de ciencias naturales se comienza a trabajar de manera independiente a partir del tercer grado; su estudio se organiza en cinco ejes temáticos, organizados de forma lineal: a) los seres vivos; b) el cuerpo humano y la salud; c) el ambiente y su protección; d) materia, energía y cambio, y e) ciencia, tecnología y sociedad.

Las asignaturas de historia, geografía y educación cívica en tercer grado se trabajan de manera modular, articulando temas sobre la comunidad, el municipio y la entidad política donde viven los nińos, pero a partir del cuarto grado, cada una de esas asignaturas se estudia de manera independiente, con su propio propósito.

Otro factor que debilita el desarrollo de la EA en el plan de educación primaria es el tiempo asignado a cada asignatura. Testimonios de profesores señalan que, en la lectura, la escritura y la formación matemática se distribuye más de $50 \%$ de las horas de trabajo y, si a ello se le suma el tiempo exigido por las actividades administrativas que se han ańadido a la tarea docente, las juntas escolares y los problemas imprevistos de los niños, que deben ser tratados con los padres, el tiempo de estudio que resta para las demás áreas es menor (Terrón, 2010).

Los planes de estudio de la Reforma de 1993 contemplaron aspectos pedagógicos que se plantean mediante la EA desde Tbilisi, 1977; por ejemplo, el énfasis en el aprendizaje permanente, superar la enseñanza informativa por una enseńanza formativa y la recomendación de 
que en todo momento, la adquisición de conocimientos esté asociada con el ejercicio de habilidades intelectuales y de reflexión... bajo la tesis de que no puede existir una sólida adquisición de conocimientos sin la reflexión sobre su sentido, así como tampoco es posible el desarrollo de habilidades intelectuales, si éstas no se ejercen en relación con conocimientos fundamentales (SEP, 1993b, p. 13).

Sin embargo, esta recomendación, en los hechos, se logra poco debido a que el recurso que los profesores tienen como base de su enseñanza y aprendizaje son los libros de texto, cuyo contenido muchas veces se aleja de las expectativas de la propuesta curricular. Además, se observa un trabajo aislado entre los especialistas que diseñan el currículo, los que diseñan los programas y quienes elaboran los libros de texto y está pendiente la organización de los contenidos de forma acorde con las demandas de nuestro tiempo, para comprender y cambiar el mundo complejo en el que vivimos, lo que implica a los especialistas y no sólo a los profesores, por amplias razones que es imposible explicar aquí; la más importantes es la disponibilidad de tiempo de los profesores de grupo.

Finalmente, en el plan de estudios de educación secundaria destaca la recomendación de vincular los contenidos de las ciencias con los fenómenos del entorno natural, para estimular en los estudiantes actitudes de responsabilidad en el cuidado de su salud y del medio ambiente (SEP, 1993c). En los programas de biología, física y química de 2006, esa orientación se presenta como una forma de fortalecer a la EA y se mantiene en el Plan de estudios 2017 (SEP, 2017a), con la limitante de que los aprendizajes ponderados son: separar basura, apagar la luz, reciclar y ahorrar agua, entre otras. El contenido se empobrece cuando se cierra la asignatura de EA y se le da fin al programa Cruzada Escolar para la Educación Básica, en 2007, pero también cuando, en la práctica, lo ambiental se reduce a los aprendizajes señalados.

Los aprendizajes que se ponderan contrastan con otras recomendaciones destacadas en el plan de estudios 2017; entre éstas, la importancia de hacer converger contenidos de otras asignaturas para comprender la relación de la sociedad con la naturaleza, los efectos de los problemas globales en el medio y buscar la sustentabilidad; incluso se plantea como importante el entendimiento de las causas de los 
conflictos territoriales actuales y sus consecuencias ambientales, sociales, culturales, políticas y económicas, así como de la producción de energía y de la industria en el mundo. Pero en la práctica, en el caso de la minería, se privilegia únicamente su relevancia económica (SEP, 2017a). Amén de otras cuestiones como las siguientes: los especialistas que diseñan los planes, programas y libros de texto han delegado a los profesores la responsabilidad de integrar los contenidos o saberes disciplinares para comprender la relación sociedad naturaleza y la complejidad del mundo y, a la par, en el plan de estudios de secundaria 2006, se resalta que los profesores delegan a los estudiantes "el trabajo de integración para relacionar los contenidos fragmentados que abordan en el tiempo de su clase" (SEP, 2006, p. 17).

\section{Materiales de apoyo educativo}

La década de los noventa fue un momento importante en el desarrollo de la EA en la educación básica; conforme a las estrategias planeadas en el Promode, al inicio del año escolar 1992-1993, la SEP inició un proceso de actualización y generación de materiales de apoyo para los profesores de primaria. En 1992, se proporcionó a la escuela primaria la Guía para el maestro sobre medio ambiente. Educación primaria (SEP, 1992). Fue un primer material educativo formal para apoyar la práctica educativa: en la introducción, se aclara que es una guía de EA que presenta a los profesores una propuesta para integrar los contenidos de ciencias naturales relacionados con el medio ambiente. La guía es pionera en el intento de articular los contenidos científicos con los problemas del ambiente que viven los alumnos, su limitación es la desvinculación con el aspecto social.

A la mencionada guía y a los programas emergentes de educación primaria desarrollados en ese mismo periodo como parte de la prueba operativa, se sumó un texto para el alumno titulado: La República mexicana: equilibrio ecológico, en cuya elaboración colaboraron la Secretaría de Desarrollo Urbano y Ecología (Sedue), la SEP y la Secretaría de Salud (SSA). Posteriormente, en 1994, en la ciudad de México, para apoyar el desarrollo de la EA en la escuela, la SEP la implementó como materia optativa en el tercer grado de secundaria; más adelante se comenzó a impartir en los estados de la República, por decisión propia de las entidades federativas. 
En la Ciudad de México, en el ciclo escolar 1997-1998, se eliminó la asignatura de EA y se impulsó en toda la educación básica el programa Cruzada escolar para la preservación y cuidado del ambiente; el programa incluyó actividades escolares y extraescolares en centros de recreación y cultura (zoológicos, museos, acuarios, planetarios, parques y viveros) que impartían programas de EA (SEP, 1997-1998); así también contempló conferencias sobre problemas de contaminación del aire, del agua, del suelo, pérdida de biodiversidad, manejo de residuos tóxicos, entre otros, que fueron dictadas por especialistas de la Universidad Nacional Autónoma de México (UNAM), la Universidad Autónoma Metropolitana (UAM) y la Secretaría del Medio Ambiente y los Recursos Naturales (Semarnat); asimismo, en otros centros los profesores recibieron talleres de reciclado, elaboración de composta, ahorro de energía, cuidado del agua y reforestación (Terrón, 2004 y 2010). Dicho programa fue eliminado en 2007.

En 1999, la SEP, a través del Programa Nacional de Actualización Permanente (Pronap), en coordinación con la Semarnap (ahora Semarnat), elaboraron un paquete didáctico para promover un curso nacional de EA en el nivel secundaria. El paquete se diseñó para la formación autodidacta del profesor, con la expectativa de que pudiera ser aprovechado por maestros tanto de educación secundaria como de primaria; desafortunadamente, fue un curso con poca demanda, en cuanto no era obligatorio y, entre la amplia oferta que ofrecía la SEP, a los profesores les interesaban más los cursos en los que eran evaluados sus alumnos: matemáticas, español y ciencias naturales.

Igualmente, a través del Pronap, se ofertaron cursos con carácter nacional en el ciclo escolar 2007-2008, entre ésos se encuentran los siguientes: Actitudes y valores para la EA en la escuela secundaria; La EA en la escuela secundaria, dirigido a maestros frente a grupo, directivos escolares y apoyo técnico pedagógico. Este curso se ubicó en el área de ciencias. La problemática ambiental desde la escuela y el salón de clases, dirigido a maestros frente a grupo del nivel primaria, con módulos sobre agua, energía y biodiversidad. Asimismo, en ese mismo ciclo escolar 2007-2008, en algunos estados del país, la SEP ofreció cursos a profesores de primaria regular e indígena, de secundaria y telesecundaria; entre esos estados, se encuentran: Hidalgo, Jalisco, Morelos y Nayarit. 
Posteriormente, en coordinación con el Centro de Capacitación para el Desarrollo Sustentables de la Ssemarnat (Cecadesu-Semarnat), las Secretarías de Educación de los estados del país se dieron a la tarea de diseñar, en forma conjunta, programas de EA para la Sustentabilidad. La idea fue impartir esos programas como asignatura estatal en el primer grado de Educación Secundaria. Los estados que impartieron esa asignatura en el ciclo escolar 2013-2014, fueron Aguascalientes, Baja California Sur, Campeche, Chiapas, Distrito Federal, Durango, Guerrero, Hidalgo, Jalisco, México, Michoacán, Morelos, Querétaro, Quintana Roo, Tabasco, Tamaulipas, Tlaxcala, Veracruz y Zacatecas (Anicasio, 2014).

\section{INVESTIGACIÓN EN EDUCACIÓN AMBIENTAL}

Desde la introducción de la EA al currículo educativo ha habido interés en la investigación en ese campo y en su desarrollo educativo. A diez ańos de haberse adoptado la EA en México por la entonces Secretaría de Desarrollo Urbano y Ecología (Sedue), en 1993 se realizó el II Congreso Nacional de Investigación Educativa promovido por el Consejo Mexicano de Investigación Educativa (Comie); en este congreso, cada diez ańos se elabora un estado del conocimiento sobre el desarrollo de la investigación en las áreas en las que trabajan los investigadores de la educación en México.

En ese segundo congreso se presentó el primer estado del conocimiento denominado La investigación educativa en los ochenta, perspectiva para los noventa, en el que, por primera vez, se analizó el estado de la investigación educativa en el contexto mexicano; en el número 13 de los estados del conocimiento enfocado en la salud, se consideró el Capítulo III Ambiente, el cual fue coordinado por las investigadoras Cristina Rivera y Rose Eisenberg Wieder de la FES Iztacala/UNAM (Rivera, et al. 1993).

En la Memoria del Foro Multinacional de Experiencias sobre la Inserción de la Dimensión Ambiental en el Currículum, no obstante que fue un foro que se realizó en el marco del Proyecto Multinacional de Educación Media y Superior (Promesup), se presenta una sección con seis ponencias, enfocadas en proyectos de intervención en EA en la educación básica y la formación valoral de docentes (PROMESUP, 1996). 
En el segundo estado del conocimiento, La Investigación Educativa en México 1992-2002, publicado por el Comie, la investigación en EA se encuentra en un campo más amplio, en el volumen 3: Educación, derechos sociales y equidad, con el Título Educación y medio ambiente. Este estado del conocimiento sobre la EA fue coordinado por Édgar González Gaudiano y Ma. Teresa Bravo Mercado, de la Facultad de Filosofía y Letras UNAM/SEP y del CESU-UNAM, respectivamente (González, 2003 y Bravo, 2003).

Por los fines de este artículo y por requerimientos del espacio, sólo quiero señalar, respecto de los estados del conocimiento, que en general, como su nombre lo indica, exponen, por una parte y de manera cuantitativa, la diversidad de temas que se desarrollan en la investigación educativa de la EA, la diversidad de sujetos que participan, de contextos y ámbitos en los que se desarrolla, de productos (tesis, libros, reportes de investigación, artículos, etcétera), enfoques teóricos metodológicos y conceptuales, así como la expansión y avance de la investigación en EA, sus retos, desafíos y pendientes.

Por otra parte, y de mucha importancia para comprender realmente el posicionamiento y peso de la EA en la investigación educativa, en la primera parte del contenido se hace una descripción reflexiva sobre cómo se ha constituido el campo de la investigación en EA en México y en América latina, cómo ha evolucionado la diversidad conceptual y social del campo, sus sesgos teóricos, conceptuales y metodológicos; sus discursos naturalistas, conservacionistas y otros discursos más propios de la región latinoamericana. Señalar los principales apuntes, recuentos y notas de estos estados del conocimiento, me dejaría en deuda con los autores y los equipos de trabajo participantes, por lo que me permito invitar a leer detenidamente el contenido de tales estados del conocimiento en las obras referidas.

Sobre el marco de referencia conceptual desde el que se posiciona a la EA en el nivel básico, puede decirse que sigue predominando en los propósitos educativos de los planes, programas y libros de texto actuales la orientación naturalista y conservacionista, lo que hace ya tres lustros González Gaudiano (2003), observaba en los libros de texto de ciencias naturales para la educación primaria en México. Pero superar la crisis ambiental requiere comprender sus verdaderas causas. Delegar la responsabilidad a la sociedad para hacer frente a los impactos de la crisis es eludir responsabilidades diferenciadas de 
los sectores sociales. Se requiere una educación crítica que nos lleve a la inclusión de los temas de desigualdad y de justicia ambiental, así como a la realización de acciones más integrales, sistémicas y de largo plazo, atendiendo las causas centrales de la problemática provocada por las industrias, en los países que más contaminan por el consumismo y las desmedidas prácticas extractivas de las riquezas naturales.

La investigación en educación ambiental para la sustentabilidad en México 2002-2012 es un tercer estado del conocimiento de la investigación en $\mathrm{EA}$, en el que dicha área se presenta de manera independiente en los estados del conocimiento promovidos por el COMIE. Fue coordinado por Édgar González Gaudiano y Miguel Ángel Arias Ortega, de la Universidad Veracruzana y de la Universidad Autónoma de la Ciudad de México (UACM) (González y Arias, 2015).

Se han realizado otros congresos y seminarios sobre la investigación en EA, entre éstos, el II Congreso Nacional de Investigación en Educación Ambiental para la Sustentabilidad "Consolidando políticas para la investigación”, organizado por la Academia Nacional de EA (ANEA) en 2011. En el balance de esos congresos se ha identificado que la EA ha venido evolucionando en el plano teórico, conceptual y metodológico, pero aún persisten, en su práctica, visiones reducidas asociadas a las orientaciones de las políticas educativas, que evitan el estudio crítico del problema de fondo, particularmente de los intereses económicos dominantes, en la diversidad de acciones que hacen crisis en el ambiente.

\section{CONTROVERSIAS EN LA PRÁCTICA DE LA EDUCACIÓN AMBIENTAL}

No obstante el enfoque naturalista en el currículo, la EA no se siguió en todas las escuelas de educación básica; muchos materiales educativos que se produjeron para fortalecer su desarrollo no llegaron a todas las zonas del país, incluso en la ciudad de México, por lo que no pudieron aprovecharse en toda su extensión (Terrón, 2010). Lo anterior es un problema recurrente de inequidad que atrasa el desarrollo educativo, debido a que los objetivos y las formas concretas que adquieren para caminar hacia los fines perseguidos se diluyen. Esa situación se manifiesta con la RIEB, pero la reforma educativa de 2017 no augura algo mejor; así lo ha constatado una investigación 
que realizo con profesores de educación primaria, en una comunidad indígena en el estado de Chiapas.

En la entrevista a seis profesores de una escuela ubicada en la comunidad señalada, el único material al que los profesores hicieron referencia fue el libro de texto, como puede observarse en las respuestas a las preguntas siguientes: "Cuándo usted enseña educación ambiental, ¿qué enseńa?”, al respecto un profesor expresó:

No es una obligación enseñarla, los alumnos tienen que aprender otras cosas, pero sabemos que este tema es importante y en las clases algunos nińos tocan los problemas que hay con el agua, nosotros les explicamos algunas cosas, pero únicamente tenemos de apoyo lo que los libros de texto nos dicen y eso es lo que se les enseña.

Otra pregunta fue "¿Cómo trabajas los contenidos sobre el cambio climático con los nińos?”, al respecto, otro profesor dijo:

Así como aparece en los libros, hay algunos temas que son difíciles de trabajar, como el del efecto invernadero, porque en el libro no aparece mucho y los niños preguntan y muchas veces, por más que uno les explique, no lo entienden.

Otro problema grave que se identifica en los libros de texto gratuitos, cuya distribución es nacional, son las ligas a Internet. Con la idea de que los estudiantes aprendan el manejo de las TIC, se incluyen links a diversas páginas para que los niños revisen contenidos en línea, sin prever que en las zonas remotas del país no se cuenta con el recurso de Internet, ni las escuelas ni los niños cuentan con computadoras. $^{3}$ Ante dicha situación, el discurso político de la EA en el currículo y su intención educativa queda en discurso e intención, debido a que difícilmente se puede llevar a la práctica de forma cabal.

\footnotetext{
${ }^{3}$ Lo señalado son testimonios expresados de diferentes maneras por los profesores de dicho nivel, con los que he trabajado en los seminarios que he impartido sobre EA, pero ha sido confirmado por otros actores que también lo viven diariamente en la trinchera; me refiero a quienes tuvieron la oportunidad de hablar de esta experiencia en la mesa "Transformación de la escuela primaria” del ciclo de conferencias La educación en México en el 2004. Viejos problemas. Nuevos actores. ¿Nuevas soluciones? (DIE/CINVESTAV, octubre 11 de 2004), en Terrón (2010). Asimismo, en la investigación que estoy realizando actualmente (2017-2019) con profesores de educación primaria en una comunidad indígena del estado de Chiapas, se revela el mismo problema.
} 
Se puede inferir que se ha hecho un manejo trivial de la EA y de los problemas del ambiente en particular; el enfoque positivista que se adopta en el currículo delinea una enseñanza y un aprendizaje dispersos, desarticulados y mecanicistas, que no inciden en el conocimiento complejo de nuestra realidad y en la construcción de valores ambientales en los estudiantes; asimismo, la reducción de los problemas ambientales a problemas ecológicos y a prácticas cotidianas remediales y descontextualizadas, no contribuye a la formación educativa que demandan nuestros tiempos (Terrón et al., 2016).

Esa trivialización se atribuye, en parte, al discurso que se ostenta en la propia política educativa, en la Ley General de Educación y en los planes nacionales de desarrollo (2001-2006, 2006-2012 y 2013-2018), porque reducen la EA al ámbito ecológico y ese discurso cruza las reformas educativas de 1993, 2006, 2009, 2011 y 2017, incluyendo los programas de actualización de docentes, los contenidos de los materiales didácticos, los textos autorizados por la SEP para la asignatura optativa sobre este tema en el tercer grado de secundaria y las asignaturas estatales de EA para la sustentabilidad.

Con el término trivial me refiero a que los problemas ambientales y contenidos relativos al campo de la EA se abordan de manera superficial y aislada en las asignaturas curriculares; se busca resolver el problema ambiental desde el marco de las ciencias naturales, cuando se sabe hace mucho tiempo que el problema ambiental rebasa los límites de la ciencia; este hecho ha sido expuesto reiteradamente, antes y después de las reformas educativas, en diversos foros y en congresos (Rivera, 1993; González, 2003; Bravo, 2003; González y Arias, 2015; Terrón, 2017, entre otros).

La organización disciplinaria del currículo fortalece la linealidad y, con ello, el estudio de lo ambiental de manera aislada y ahistórica; reduce el problema ambiental, propone soluciones por partes, en forma desvinculada, y omite los elementos integradores y emergentes del problema en su conjunto como unidad, con sus sentidos y significados. Al reducir el problema a acciones irreflexivas, remediales y conservacionistas de protección, descontaminación, mitigación, se obstaculizan las soluciones integrales que incluyen la justicia social, el respeto a la tierra, a la seguridad humana y los derechos humanos a una vida digna y a un ambiente saludable, así como al desarrollo de un pensamiento crítico, complejo y relacional, con 
visión planetaria, fortalecido en la transdisciplinariedad, en tanto que, a la luz de algunos planteamientos de Nicolescu (1996) sobre la transdisciplinariedad, se parafrasea que la EA

aborda un problema educativo complejo y sistémico, revoluciona el proceso de pensar en la comprensión y transformación de la crisis ambiental, rescata en ese proceso de conocimiento y de transformación, no sólo el problema ecológico, sino también al sujeto, su subjetividad y el bien común. En su proceso educativo propicia operaciones complejas del pensamiento mediante la convergencia cognitiva de las ciencias naturales, ciencias sociales, ciencias humanas y arte, en las que confluyen la historia, conocimientos bio-físicos, sociales, económicos, culturales, educativos, valores y espiritualidad. Esclarece el vínculo teórico de ese pensamiento con la práctica para coadyuvar al posicionamiento crítico y de transformación de los sujetos, respecto de una realidad ambiental dinámica y en continua interacción, que da lugar a fenómenos emergentes e incertidumbre, con consecuencias ambientales de riesgo para nuestros pueblos.

Mediante la EA se facilita el desarrollo de un pensamiento relacional, analítico, complejo, integrador y crítico que permite resignificar el sentido y significado del conocimiento y del aprendizaje en el cuidado planetario, en un ambiente educativo afectivo, horizontal de diálogo, inclusión, respeto, reconocimiento y respeto entre los sujetos con culturas diversas, el diálogo de saberes (científicos, tradicionales, populares), la participación colaborativa que, entre otras cosas, favorece la acción comunicativa entre el profesor y los estudiantes, la escuela, la comunidad y los otros, aportando y compartiendo a un proyecto conjunto de cuidar y preservar los sistemas de vida, el bienestar humano y nuestro planeta (Leff, 2003 y 2015 y Terrón, 2017, entre otros).

La incorporación de la EA en el currículo de la educación básica implicó todo un proceso y trabajo de organización de parte de la SEP; pueden observarse en la reforma de 1993 grandes expectativas de cambios en la educación formal, con el inconveniente de que no se integran en la práctica, al no traducirse, en su conjunto, en los programas, en los libros de texto, ni en la formación y actualización de profesores. Los fines educativos presentes en el currículo se van dilu- 
yendo y los contenidos ambientales tienen la influencia del mandato Presidencial de 1986, de incorporar en la pedagogía ecológica la materia de ecología en la formación inicial de profesores y temas ecológicos en los libros de texto, así como de la Ley Federal de Educación, ya que dichos contenidos se concentran en las ciencias naturales.

El desarrollo de la EA en la educación básica se realiza con visiones que están rebasadas por la complejidad de la crisis ambiental y la dinámica de la vida; persisten, en los contenidos, las controversias educativas evidenciadas en el diagnóstico que realizó el Conalte, en1989; ese balance no ha variado mucho en el momento actual. Las recomendaciones del Acuerdo Nacional para la Modernización Educativa y, posteriormente, del Promode, se enfocaron mejor en los planes de estudio de 1993, incluso en los programas de las asignaturas, que en los libros de texto, materiales educativos y cursos para docentes.

Las reformas y programas educativos 2004, 2006, 2009, 2011 y 2017 de la educación básica conservan las directrices acerca del ambiente en los planes, programas y libros de texto y en las mismas asignaturas que en la Reforma de 1993, pero hace falta el desarrollo de un pensar y actuar con visión integradora y planetaria, que tome el objeto de la EA como campo complejo de convergencia e interacción y, mediante una práctica educativa crítica, pueda contribuir al cambio de formas de socialización del conocimiento, de los aprendizajes y a la transformación social que se plantea a través de dicha educación.

Con los objetivos por competencias, los estándares de aprendizaje y los aprendizajes esperados promovidos en las últimas reformas educativas se apuntaló una educación básica desarrollista, más que formativa; la constante es un contrasentido, en cuanto se priorizan saberes fragmentados y el contenido privilegiado para realizar los ideales curriculares soslaya aprendizajes de la educación y formación integrales, concebidos en el planteamiento curricular.

Lo ambiental se objetiva en actividades remediales de conservación de los recursos naturales, de separación de basura, reciclaje y mitigación (SEP, 2017a). Así, se evita la reflexión crítica de los valores materialistas, del papel de la economía, de la política y de la cultura que hacen crisis en el ambiente y su impacto socioambiental recursivo. Está ausente la responsabilidad en la crisis ambiental de la economía, la política, la cultura del progreso, de algunas tecnologías y, su ética, es una omisión que obstaculiza el entendimiento de la 
complejidad del mundo, la toma de conciencia crítica y el posicionamiento libre, responsable y comprometido de los estudiantes y la sociedad en la búsqueda del bienestar planetario.

No obstante que en el Modelo educativo de 2017 se alude a términos como complejidad e interdisciplina, la lógica de organización curricular por asignaturas es lineal, no se establecen correlaciones entre conocimientos de las asignaturas para comprender los problemas ambientales de estudio y sus alternativas de solución con perspectiva integral, excepto en la asignatura de geografía, con el problema de que la responsabilidad de la integración de conocimientos se le deja al docente; en otras palabras, falta mucho por hacer. Los especialistas que elaboran los libros de texto y los materiales educativos para apoyar la docencia y a los profesores no se han sentado a trabajar en la organización de los contenidos con un enfoque complejo e interdisciplinario, ni se ha atendido la formación de los profesores en ese sentido.

Así pues, a 37 ańos de realizada la Consulta Nacional para la Modernización de la Educación y 25 de haberse implementado la Reforma educativa de 1993, se observa que la organización lineal de los contenidos, la fragmentación del conocimiento y los valores instrumentales que se privilegian en el currículo ha contribuido a que el enfoque integral que se enfatiza en el discurso se desvanezca en cada asignatura, ya que pese a la transversalidad de contenidos ambientales, se parcelan y se estudian desarticulados. Es, pues, importante otorgar a la EA un espacio curricular propio, como un campo educativo integrador de esos conocimientos, pero diferenciado, a la vez, y acorde con la diversidad ecosistémica y sociocultural de los contextos de los estudiantes.

\section{CONSIDERACIONES FINALES}

El estudio del desarrollo de la EA en la educación básica en México ha sido una preocupación anterior y posterior a su incorporación en los currículos educativos; las investigaciones de Chamizo (1990), De Alba (1993), de Jiménez y Hernández (1988), así como los estados del conocimiento que se realizan cada diez años en el marco del desarrollo del Congreso Nacional de Investigación Educativa y otros son una muestra de ello.

La forma como se ha venido configurando la EA reafirma la tesis de que la institución educativa contribuye, de alguna manera, en la 
construcción de sentido de una cultura y gestión ambiental en el ámbito escolar. La institución educativa es una mediación sustantiva del proceso de EA que se desarrolla en ella, comenzando con la política educativa, la renovación de planes de estudio con un enfoque ambiental, de cursos de actualización de profesores, de programas y tareas específicos que se han organizado para desarrollar de mejor manera la EA en el ámbito escolar (Terrón, 2010).

La política educativa en México influyó ampliamente en la inserción de la EA en la escuela; sin embargo, queda un largo camino por andar, debido a que la forma transversal en que tal inserción se ha llevado a cabo, aunque representa un avance, no es suficiente. No se alcanza la integración del conocimiento que se necesita en la EA para lograr una visión globalizadora e integradora de la realidad ambiental local y planetaria que permita a los estudiantes una mayor resignificación del hecho educativo y de los aprendizajes en la interpretación de los problemas ambientales, de su transformación y el cambio en su forma de relacionarse para transitar hacia una convivencia armónica con la Tierra, sus congéneres y consigo mismos.

Si bien un logro importante en el desarrollo de la EA ha sido la inserción del estudio de problemas ambientales en las asignaturas del currículo, la formulación y desarrollo de cursos con docentes; la producción de materiales didácticos, libros, artículos, tesis y la presentación de ponencias en eventos académicos persiste el reto de superar el currículo positivista, de promover un currículo crítico humanista y, dentro de éste, dar a la EA un espacio curricular que en su proceso educativo aporte a los estudiantes elementos para comprender la complejidad de la dinámica de la vida planetaria, para afrontarla digna e interculturalmente, de manera justa, solidaria, responsable, colaborativa y respetuosa, en el marco de su diversidad biológica y sociocultural.

En las reformas educativas posteriores a la de 1993, cuya finalidad fue mejorar "la calidad de la educación básica", no hubo interés por hacer coherente la teoría con la práctica de la EA y se empobreció su discurso teórico, metodológico y ético. El esfuerzo de la SEP se desvaneció al priorizar una educación técnica y prácticas remediales de lo ambiental, bajo la idea de que eso soluciona el problema, olvidando que éste es más amplio. Coadyuvar en su disminución plantea cambios en la economía, la cultura, la política, en la educación y 
en la ética como una posibilidad para conocer, actuar y relacionarse de otra manera.

De hecho, se quieren modificaciones de fondo: se necesita que el currículo vaya más allá de la visión instrumental técnica que sostiene a los organismos económicos; se requiere adoptar perspectivas educativas humanistas, integrales, significativas y profundas que permitan superar el reduccionismo mecanicista, el individualismo, la competencia, el egoísmo y la indiferencia en el que han caído la práctica educativa de la educación general y las relaciones sociales e interpersonales en el mundo.

La EA es una alternativa para el cambio, pero es necesario desarrollarla en su profundidad, como una educación humanista descolonizadora de la cultura materialista, consumista y reduccionista de la vida, apoyándose en la constitución de una conciencia crítico reflexiva y compleja, que posibilite que los sujetos comprendan y tomen postura sobre los cambios necesarios para superar la crisis ambiental, incluyendo prácticas, valores y formas de vida sustentados en el buen vivir.

Se necesita, también, que los docentes cuenten con formación en el campo de la EA, incluyendo la formación respecto de lo humano, que les proporcione una cultura teórica, epistemológica y pedagógica que haga factible el vínculo teoría-práctica, la comprensión de los problemas ambientales en su complejidad, el autoconocimiento de los sujetos en el marco del proceso en el cual han construido sus valores sobre la vida y su actuación en ella, tomando conciencia crítica de las relaciones que motivan esos valores y su incidencia en la crisis ambiental, así como de la ética indispensable para transitar hacia una vida planetaria armónica.

Es importante, además, que en el repensar la EA y su proceso formativo se tome en cuenta la multidimensionalidad y complejidad del fenómeno educativo y de la crisis ambiental como un todo; la diversidad sociocultural y de las comunidades de los alumnos en sus contextos; los conocimientos y forma propicia para que los estudiantes aprendan el arte de vivir y convivir con calidad humana y de vida, en armonía con la tierra, con los otros seres humanos, con otras culturas y consigo mismos, buscando construir una cultura ambiental fuerte, cimentada en una ética humanista e intercultural; ello, teniendo en cuenta que lo que se estará formando son los nuevos ciudadanos de México y del mundo. 


\section{REFERENCIAS BIBLIOGRÁFICAS}

Anicasio, J. (2014). Análisis de programas de estudio de educación ambiental para la sustentabilidad en la educación secundaria.(Tesis de maestría). Universidad Pedagógica Nacional, Ajusco, México.

Bravo, M. T. (2003). La investigación en educación y medio ambiente. En M. Bertely (Coord.), La investigación educativa en México 1992-2002, Educación, derechos sociales y equidad, tomo 3, Educación y medio ambiente (pp. 277-369). México: Comie.

Chamizo, O. (1990). Los contenidos ambientales en el nivel de educación preescolar. Revista Cero en conducta, 17(5), 41-44.

Conalte (1992). Educación ambiental en la educación básica. En J. E. Grajeda (Coord.), Memoria: Foro internacional para la incorporación de la dimensión ambiental en la educación media superior (pp. 75-84). México: OEA/SEP/SEIT/DGETA.

De Alba, A. et al. (1993). El libro de texto y la cuestión ambiental. Los contenidos ecológicos en el curriculum de primaria. México: Sedesol/CESU-UNAM.

Estermann, J. (2012). Crisis civilizatoria y vivir bien. Polis. Revista de la Universidad Bolivariana, 11(32), 149-174.

Diario Oficial de la Federación (1992). Acuerdo Nacional para la Modernización de la Educación Básica. Recuperado de http://www.sep.gob.mx/work/ models/sep1/Resource/b490561c-5c33-4254-ad1c-aad33765928a/07104.pdf

González Gaudiano, É. et al. (1997). Educación ambiental. Historia y conceptos a veinte años de Tbilisi. México: Sistemas Técnicos de Edición.

González Gaudiano, É. (2003). Atisbando la construcción conceptual de la educación ambiental en México. En M. Bertely (Coord.), La investigación educativa en México 1992-2002, Educación, derechos sociales y equidad, tomo 3, Educación y medio ambiente (pp. 243-275). México: Comie.

González Gaudiano, É., y Arias, M. Á. (Coords.) (2015). La investigación en educación ambiental para la sustentabilidad en México (2002-2011). México: ANUIES: COMIE.

Jiménez, P., y Hernández, S. (1988). Análisis de la educación ambiental en la educación media básica. Revista Cero en conducta, 10(3), 16-22.

Leff, E. (2003). Pensar la complejidad ambiental. En E. Leff (Coord.), La complejidad ambiental (pp. 7-53). México: Siglo XXI.

Leff, E. (2015). De la multi a la inter y la transdisciplina: especializaciones e hibridaciones entre ciencias sociales, ciencias ambientales y ecología humana. En A. Argueta y G. Peimbert (Coords.), La ruptura de las 
fronteras imaginarias o de la multi a la transdisciplina (pp. 41-59). México: UNAM/CRIM/Siglo XXI.

Ley General de Educación (1993). Capítulo I de las disposiciones generales, Artículo 7o punto (XI), Última Reforma DOF 19-01-2018. México: Cámara de Diputados del H. Congreso de la Unión.

Morin, E., y Delgado C. (2016). Reinventar la educación. Abrir caminos a la metamorfosis de la humanidad. México: Multiversidad Mundo Real.

Nicolescu, B. (1996). La transdisciplinariedad. Manifiesto. México: Multiversidad Mundo Real Edgar Morin.

Olivé, L. (2015). El aprovechamiento social del conocimiento tradicional: un problema transdisciplinario. En A. Argueta y G. Peimbert (Coords.), La ruptura de las fronteras imaginarias o de la multi a la transdisciplina (pp. 80-103). México: UNAM/CRIM/Siglo XXI

Poder Ejecutivo Federal (2001). Plan Nacional de Desarrollo 2001-2006. México: Poder Ejecutivo Federal.

Poder Ejecutivo Federal (1996). Programa de Desarrollo Educativo 19952000. México: Poder Ejecutivo Federal.

Poder Ejecutivo Federal y SEP (1989). Programa nacional para la modernización educativa 1989-1994. México: SEP.

Promesup (1996). Foro multinacional de experiencias sobre la inserción de la dimensión ambiental en el curriculum. México: OEA/SEP/SEIT/DGETA.

Rivera, C. et al. (1993). Ambiente. En R. Eisenberg (Coord.), La investigación educativa en los ochenta, perspectiva para los noventa. Estados del conocimiento 13, (cap. III). 20 Congreso Nacional de Investigación Educativa. México: Comité organizador/SNTE.

Semarnat (s/f). Antecedentes. Recuperado de www.semarnat.gob.mx

SEP (1992). Guía para el maestro. Medio ambiente y educación primaria. México: SEP. SEP (1993a). Artículo $3^{\circ}$ Constitucionaly Ley General de Educación. México: SEP. SEP (1993b). Plan y programas de Estudio de Educación Básica/Primaria. México: SEP.

SEP (1993c). Plan y programas de Estudio de Educación Básica/Secundaria. México: SEP.

SEP (1997-1998). Cruzada escolar para la preservación y cuidado del ambiente. México: Subsecretaría de servicios educativos para el Distrito Federal. Coordinación de programas complementarios. Dirección de enlace normativo y apoyo administrativo.

SEP (2001). Programa nacional de educación 2001-2006. México: SEP.

SEP (2004). Programa de educación preescolar 2004. México: SEP. 
SEP (2006). Plan de estudios 2006. Educación Básica Secundaria. México: SEP.

SEP (2009). Plan de Estudios 2009. Educación Básica Primaria. México: SEP. SEP (2011). Plan de estudios 2011. Educación Básica. México: SEP.

SEP (2017a). Plan y programas de estudio, orientaciones didácticas y sugerencias de evaluación. México: SEP

SEP (2017b). Estadística del Sistema Educativo Mexicano ciclo escolar 2016-2017. Recuperado de www.inee.edu.mx/mapa2015/pdfestados/ prontuario2015.pdf

Terrón, E. (2004). La educación ambiental en la educación básica, un proyecto inconcluso. Revista Latinoamericana de Estudios Educativos, XXXIV(4), 107-164.

Terrón, E. (2010). Educación Ambiental. Representaciones sociales y sus implicaciones educativas. Ciudad de México: UPN.

Terrón , E., Sánchez, M. S., y Bahena, D. G. (2016). El pensamiento de los jóvenes sobre el cambio climático: el camino pendiente de la educación ambiental. En A. Espejel y R. Calixto (Coords.), Educación, jóvenes y ambiente (pp. 73-101). México: UAT/UPN.

Terrón, E. (2017). Diálogo entre educación ambiental, complejidad y pedagogía ambiental para entender el mundo hoy y transformarlo integralmente. En J. Reyes y E. Castro (Coords.), Travesias y dilemas de la pedagogía ambiental en México (pp. 11-38). México: Editorial Universitaria.

UNESCO (1977). Tendencias de la educación ambiental. Francia: UNESCO.

UNESCO (1978). Conferencia Intergubernamental sobre educación ambiental. Informe final (Tbilisi, 14-26 de octubre de 1977). Francia: UNESCO.

UNESCO (1980). La educación ambiental. Las grandes orientaciones de la Conferencia de Tbilisi. Francia: UNESCO. 